\title{
ON WEAKLY COUNTABLY DETERMINED BANACH SPACES
}

\author{
SOPHOCLES MERCOURAKIS
}

\begin{abstract}
For a topological space $X$, let $C_{1}(X)$ denote the Banach space of all bounded functions $f: X \rightarrow \mathbf{R}$ such that for every $\varepsilon>0$ the set $\{x \in$ $X:|f(x)| \geq \varepsilon\}$ is closed and discrete in $X$, endowed with the supremum norm. The main theorem is the following: Let $L$ be a weakly countably determined subset of a Banach space; then there exist a subset $\Sigma^{\prime}$ of the Baire space $\Sigma$, a compact space $K$, and a bounded linear one-to-one operator $T: C(L) \rightarrow C_{1}\left(\Sigma^{\prime} \times K\right)$ that is pointwise to pointwise continuous. In the case where $L$ is weakly analytic, $\Sigma^{\prime}$ can be replaced by $\Sigma$. This theorem is connected with the basic result of Amir-Lindenstrauss on WCG Banach spaces and has corresponding consequences such as: the representation of Gulko (resp. Talagrand) compact spaces as pointwise compact subsets of $C_{1}\left(\Sigma^{\prime} \times K\right)$ (resp. $C_{1}(\Sigma \times K)$ ) (a compact space $\Omega$ is called Gulko or Talagrand compact if $C(\Omega)$ is WCD or a weakly $K$-analytic Banach space); the characterization of WCD (resp. weakly $K$-analytic) Banach spaces $E$, using one-to-one operators from $E^{*}$ into $C_{1}\left(\Sigma^{\prime} \times K\right)$ (resp. $\left.C_{1}(\Sigma \times K)\right)$; and the existence of equivalent "good" norms on $E$ and $E^{*}$ simultaneously.
\end{abstract}

Introduction. The purpose of the present paper is the study of $K$-analytic and, more generally, countably determined Banach spaces in the weak topology. As Talagrand proved in [21] the well-known class of weakly compactly generated (WCG) Banach spaces consists of weakly $K$-analytic spaces. Moreover, Talagrand proved that these classes of Banach spaces are distinct $[\mathbf{2 1}, \mathbf{2 3}]$.

The classical Banach space $C_{0}(\Gamma)=\{f: \Gamma \rightarrow \mathbf{R}$ : for every $\varepsilon>0$ the set $\{\gamma \in$ $\Gamma:|f(\gamma)| \geq \varepsilon\}$ is finite $\}$ has a basic role for the structure of weakly compact subsets of Banach spaces, in view of the classical theorem of Amir-Lindenstrauss [1]: if $E$ is a WCG Banach space, then there exist a set $\Gamma$ and a bounded linear one-toone operator $T$ from $E$ into $C_{0}(\Gamma)$. It is an immediate consequence of this result that every weakly compact subset of a Banach space is homeomorphic to a weakly compact subset of $C_{0}(\Gamma)$.

The proof of the Amir-Lindenstrauss (abbreviated A-L) Theorem relies on the method of "long" sequences of projections of Banach spaces, which is a compactness argument.

Vasak [24] and Gulko [9] working independently, extended the method of projections of A-L to the wider class of weakly countably determined Banach spaces (WCD). Especially Gulko replaced the (functional) projections of Banach spaces by (topological) retractions of compact spaces.

The works of Vasak and Gulko leave open the following question: is there an A-L type theorem for WCD spaces? In this paper we answer affirmatively this question by introducing a new class of spaces corresponding to the spaces $C_{0}(\Gamma)$.

Received by the editors October 1, 1985 and, in revised form, February 26, 1986.

1980 Mathematics Subject Classification (1985 Revision). Primary 46B20, 54H05. 
Definition 1.1. Let $X$ be a topological space. We set $C_{1}(X)=\{f: X \rightarrow \mathbf{R}: f$ is bounded and for every $\varepsilon>0$ the set $\{t \in X:|f(t)| \geq \varepsilon\}$ is closed and discrete in $X\}$. It is clear that $C_{1}(X)$ is a Banach space, being a closed linear subspace of $l^{\infty}(X)$.

We notice that

(a) For a subset $A$ of $X, A$ is closed and discrete in $X$ if and only if $A^{\prime}$, the derived set of $A$, is empty,

(b) $C_{0}(X) \subseteq C_{1}(X)$, and if $f \in C_{1}(X)$ then $f \mid \Omega \in C_{0}(X)$ for all compact subsets $\Omega$ of $X$, and

(c) if $A$ is compact (resp. closed and discrete) in $X$, then $C_{0}(A)$ (resp. $\left.l^{\infty}(A)\right)$ is a complemented subspace of $C_{1}(X)$. In particular, if $X$ is compact (resp. discrete) then $C_{1}(X)=C_{0}(X)$ (resp. $\left.C_{1}(X)=l^{\infty}(X)\right)$.

(d) If $X$ is Lindelöf then it is easy to see that for every $f \in C_{1}(X)$ the set $\{t \in X: f(t) \neq 0\}$ is at most countable (see also Lemma 4.10).

In $\S 1$ we study the pointwise compact subsets of the space $C_{1}(X)$ for a countably determined or a $K$-analytic topological space $X$. The main result (Theorem 1.2) is that if $X$ is countably determined (resp. $K$-analytic) then every pointwise compact subset of $C_{1}(X)$ is Gulko (resp. Talagrand compact); see Definition 0.3. In $\S 2$ we prove an A-L type theorem which is the main result of the paper.

THEOREM 2.5. Let $L$ be a weakly countably determined subset of a Banach space $E$ and $\alpha$ the least cardinal such that $d(L) \leq 2^{\alpha}$. Then there exists a nonempty subset $\Sigma^{\prime}$ of $\Sigma$ and a bounded linear one-to-one operator $T: C(L) \rightarrow C_{1}\left(\Sigma^{\prime} \times\{0,1\}^{\alpha}\right)$ that is pointwise to pointwise continuous. Further when $L$ is weakly $K$-analytic the set $\Sigma^{\prime}$ can be chosen to be $\Sigma$.

This result, which is fundamental for WCD and weakly $K$-analytic Banach spaces (as will be clear from its applications in $\S \S 3$ and 4), is obtained by improving and strengthening the techniques of Gulko.

$\S 3$ contains topological consequences of Theorem 2.5. The most important are representations and topological characterizations of Gulko and Talagrand compact spaces (Theorems 3.1-3.3 and Remark 3.4.1). Our representation of Gulko compact spaces implies immediately the main result of Gulko in [9] that every Gulko compact space is Corson compact.

$\S 4$ contains functional analytic consequences of Theorem 2.5: namely we characterize WCD and weakly $K$-analytic Banach spaces $E$ using the set-theoretic ordering of compact subsets of a separable metric space and operators from $E^{*}$ into spaces of the form $C_{1}(X)$ for countably determined or $K$-analytic topological spaces $X$ (Theorems 4.1 and 4.2). We prove that the fundamental geometric property of strict convexity of $C_{0}(\Gamma)[\boldsymbol{7}]$ is preserved by $C_{1}(X)$ where $X$ is countably determined (Theorem 4.5). Finally we strengthen a renorming theorem of Vasak [24] proving that every WCD Banach space admits an equivalent locally uniformly convex and smooth norm whose dual norm is strictly convex (Theorem 4.8).

ACKNOWLEDGMENT. I would like to express my special thanks to Professor S. Negrepontis who originally introduced me to the subject treated here. I also thank Professor S. Argyros for useful conversations on some parts of this paper, and Professor G. Koumoullis on the subject of descriptive set theory and for valuable help in the preparation of the present paper. 
0. Preliminaries. By the term space we mean a Hausdorff completely regular topological space.

For a space $X, w(X), d(X)$ and $\mathcal{K}(X)$ denote the (topological) weight, the density character and the set of nonempty compact subsets of $X$.

If $X$ is a space, $C(X)$ is the Banach space of continuous bounded real-valued functions on $X$ with supremum norm. The pointwise topology on $C(X)$ is determined by the requirement: a net $\left(f_{i}\right)_{i \in I}$ in $C(X)$ converges in the pointwise topology to some $f \in C(X)$ if $\lim f_{i}(x)=f(x)$ for all $x \in X$.

For every metric space $(M, d)$ we define the Hausdorff metric $d_{H}$ on $\mathcal{K}(M)[8$, problem 4.5.22] as follows:

$$
d_{H}(A, B)=\sup \{d(a, B), d(b, A): a \in A, b \in B\} .
$$

It is proved that if $(M, d)$ is complete (resp. separable or compact) then $\left(K(M), d_{H}\right)$ is complete (resp. separable or compact) (see [8, problems 4.5.22(d), 2.7.20(b) and $3.12 .26(\mathrm{a})])$. It can also be proved that if $K$ is a compact subset of $K(M)$ then the set $U\{\Omega: \Omega \in K\}$ is a compact subset of $M[\mathbf{5}$, Theorem 3.1].

We denote by $\Sigma=\omega^{\omega}$ the set of infinite sequences of natural numbers (where $\omega$ denotes the set of natural numbers), endowed with the product topology. As is well known, $\Sigma$ is a Polish space (that is, homeomorphic to a complete separable metric space), and every Polish space is a continuous image of $\Sigma$ [17, Corollary 2.4.3]. For every $\rho, \sigma \in \Sigma$, we define $\sigma \leq \rho$ if $\sigma(n) \leq \rho(n)$ for all $n \in \omega$. It is clear that for every $\rho \in \Sigma$ the set $\Sigma(\rho)=\{\sigma \in \Sigma: \sigma \leq \rho\}$ is a compact subset of $\Sigma$.

$S$ denotes the set of finite sequences of natural numbers. For $s \in S$ we denote by $|s|$ the length (i.e. the domain) of $s$. For $s \in S$ and $\sigma \in \Sigma$ (resp. $t \in S$ with $|t| \geq|s|$ ) we write $s<\sigma$ (resp. $s<t$ ) if $s$ is equal to the first $|s|$ terms of the sequence $\sigma$ (resp. $t$ ). If $\sigma \in \Sigma$ and $n \in \omega, \sigma \mid n$ denotes the finite sequence of the first $n$ terms of $\sigma$.

For two spaces $X, Y$ a mapping $f: X \rightarrow \mathcal{K}(Y)$ is said to be upper semicontinuous (usco) $[\mathbf{1 7}, \mathbf{2 1}]$, if for every $x \in X$ and every open set $V$ of $Y$ containing $f(x)$ the set $\{z \in X: f(z) \subseteq V\}$ is a neighborhood of $x$. It is easy to see that for every compact subset $K$ of $X$ the set $f(K)=\bigcup\{f(x): x \in K\}$ is a compact subset of $Y$.

Proposition $0.1[\mathbf{2 1}]$. Let $K$ be a compact space and $A \subseteq K$. Then the following are equivalent.

(a) There exists a sequence $\left(A_{n}\right)$ of compact subsets of $K$, such that for every $x \in A$, there exists a subset $D \subseteq \omega$ such that $x \in \bigcap_{n \in D} A_{n} \subseteq A$.

(b) There exist a family $\left(B_{s}\right)_{s \in S}$ of compact subsets of $K$ and a subset $\Sigma^{\prime} \subseteq \Sigma$ such that $A=\bigcup_{\sigma \in \Sigma^{\prime}} \bigcap_{s<\sigma} B_{s}$.

(c) There exist a subset $\Sigma^{\prime} \subseteq \Sigma$ and an usco mapping $f: \Sigma^{\prime} \rightarrow \mathcal{K}(A)$ such that $f\left(\Sigma^{\prime}\right)=\bigcup_{\sigma \in \Sigma^{\prime}} f(\sigma)=A$.

(d) $A$ is a continuous image of a closed subset of some space of the form $\Sigma^{\prime} \times K$ where $\Sigma^{\prime} \subseteq \Sigma$.

If a space $A$ satisfies some of the above equivalent conditions in a compact space $K$ that contains $A$ homeomorphically then $A$ is said to be countably determined. Conditions (b), (c) and (d) remain equivalent if $\Sigma^{\prime}$ is replaced by $\Sigma$. $A$ is said to be $K$-analytic if some of the conditions (b), (c), (d) are satisfied for $\Sigma^{\prime}=\Sigma$. It is clear that every $K$-analytic space is countably determined. 
We note that (see [17 and $\mathbf{2 1}]$ ) every countably determined space is Lindelöf, and that the class of these spaces is closed under continuous images, closed subsets, countable products, intersections, and unions. The same is true for the class of $K$-analytic spaces.

DEFINITION $0.2[\mathbf{7}, \mathbf{2 1}, \mathbf{2 4}]$. A (real) Banach space $E$ is said to be

(a) weakly compactly generated (WCG) if it contains a weakly compact total subset,

(b) weakly $K$-analytic (resp. weakly countably determined-WCD) if $E$ in its weak topology is $K$-analytic (resp. countably determined). It follows that every weakly $K$-analytic Banach space is WCD.

M. Talagrand has proved [21, Theorem 3.2] that every WCG Banach space is weakly $K$-analytic.

Definition $0.3[\mathbf{2}, \mathbf{7}, \mathbf{1 3}, \mathbf{1 6}]$. Let $K$ be a compact space. Then,

(a) $K$ is called an Eberlein compact, if $K$ is homeomorphic to a weakly compact subset of a Banach space,

(b) $K$ is called a Talagrand (resp. Gulko) compact if $C(K)$ is weakly $K$-analytic (resp. WCD),

(c) $K$ is called a Corson compact if for some set $\Gamma, K$ is homeomorphic to a subset of $\Sigma\left(\mathbf{R}^{\Gamma}\right)=\left\{x \in \mathbf{R}^{\Gamma}: \operatorname{supp}(x)\right.$ is countable $\}$, where $\operatorname{supp}(x)=\left\{\gamma \in \Gamma: x_{\gamma} \neq 0\right\}$ for every $x \in \mathbf{R}^{\Gamma}$, and of course $\mathbf{R}^{\Gamma}$ has the cartesian product topology. The space $\Sigma\left(\mathbf{R}^{\Gamma}\right)$ is called the $\Sigma$-product of the real line.

It must be noted that $K$ is Eberlein compact if and only if $C(K)$ is WCG [19, Corollary 3.4].

M. Talagrand has constructed a Talagrand compact space that is not an Eberlein compact [21], and a Gulko compact that is not a Talagrand compact [23].

S. P. Gulko [9] has proved that every Gulko compact space is a Corson compact space.

For the theory of WCG, weakly $K$-analytic and WCD Banach spaces we refer to $[\mathbf{7}, \mathbf{1 6}, \mathbf{2 1}$ and $\mathbf{2 4}]$.

Given a set $\Gamma, l^{\infty}(\Gamma)$ is the Banach space of all bounded real valued functions defined on $\Gamma$ under the supremum norm; $C_{0}(\Gamma)$ denotes the (closed linear) subspace of $l^{\infty}(\Gamma)$ consisting of all $f$ such that for every $\varepsilon>0$ there exists a finite subset $F_{\varepsilon}$ of $\Gamma$ with $|f(\gamma)|<\varepsilon$ for all $\gamma \notin F_{\varepsilon}$.

The ordinals are defined in such a way that an ordinal is the set of smaller ordinals. A cardinal is an ordinal not in one-to-one correspondence with any smaller ordinal. The least cardinal strictly greater than $\alpha$ is denoted by $\alpha^{+}$. The cardinality of a set $A$ is denoted by $|A|$ and the cardinality of the family $P(A)$ of all subsets of $A$ by $2^{|A|}$.

In most cases, the notation and terminology are from $[\mathbf{1 6}, \mathbf{2 1}, 14$ and 2].

1. The Banach space $C_{1}(X)$. In this section we introduce the class of Banach spaces of the form $C_{1}(X)$, where $X$ is a space, and study their pointwise compact subsets when $X$ is countably determined.

We shall prove the following

THEOREM 1.2. If $X$ is a countably determined (resp. $K$-analytic) space, then every pointwise compact subset of $C_{1}(X)$ is Gulko compact (resp. Talagrand compact). 
For the proof we introduce two classes of spaces $F^{\infty}$ and $D^{\infty}$ (Definition 1.3) and prove some of their properties (Lemmas 1.4-1.6).

DeFinition 1.3. (i) Let $M$ be a separable metric space and $F=\left\{\Gamma_{K}: K \in\right.$ $\mathcal{K}(M)\}$ a family of sets with the following property: if $K_{1}, K_{2} \in \mathcal{K}(M)$ and $K_{1} \subseteq K_{2}$ then $\Gamma_{K_{1}} \subseteq \Gamma_{K_{2}}$. We define the topological space $F^{\infty}(M)$ as the space $\Gamma \cup\{\infty\}$ where $\Gamma=\bigcup\left\{\Gamma_{K}: K \in \mathcal{K}(M)\right\}$ and $\infty$ is a point not in $\Gamma$, endowed with the following topology: each point in $\Gamma$ is isolated, while a neighborhood base for $\infty$ consists of the sets $(\Gamma \backslash A) \cup\{\infty\}$, where $A \subseteq \Gamma$ is such that $\left|A \cap \Gamma_{K}\right|<\omega$ for all $K \in \mathcal{K}(M)$.

(ii) Let $X$ be a topological space and $\infty$ a point not in $X$. We define the topological space $D^{\infty}(X)$ as the space $X \cup\{\infty\}$ endowed with the following topology: each point in $X$ is isolated, while a neighborhood base for $\infty$ consists of the sets $(X \backslash A) \cup\{\infty\}$, where $A$ is closed and discrete in $X$.

It is clear that $F^{\infty}(M)$ and $D^{\infty}(X)$ are completely regular and Hausdorff and that the sets $\Gamma_{K} \cup\{\infty\}$ are compact in $F^{\infty}(M)$.

LEMMA 1.4. $F^{\infty}(M)$ is countably determined and, when $M$ is complete, $K$ analytic.

PROOF. Let $K$ be a compactification of $F^{\infty}(M)$ and let $\left\{V_{n}: n \in \omega\right\}$ be a countable base for the topology of $K(M)$ with the Hausdorff metric (see $\S 0$ ). Set $B_{n}=\operatorname{cl}_{K}\left(\bigcup_{A \in V_{n}} \Gamma_{A}\right)$ for $n<\omega$. It is clear that each $B_{n}$ is compact and so it is enough to show that for every $x \in F^{\infty}(M)$ there exists $N \subseteq \omega$ such that $x \in \bigcap_{n \in N} B_{n} \subseteq F^{\infty}(M)$ (see Proposition 0.1).

Let $A \in \mathcal{K}(M)$ such that $x \in \Gamma_{A}$ and set $N=\left\{n \in \omega: A \in V_{n}\right\}$ so that $x \in \bigcap_{n \in N} B_{n}$. Now suppose (for the purpose of a contradiction) that there exists $y_{0} \in \bigcap_{n \in N} B_{n} \backslash F^{\infty}(M)$. Then there exists a neighborhood $U_{1}$ of $y_{0}$ in $K$ such that $\infty \notin \bar{U}_{1}$. Let $\left\{n_{k}: k \in \omega\right\}$ be an enumeration of $N$. We shall construct inductively a nontrivial sequence $\left\{y_{k}\right\}$ in $U_{1}$ and a sequence $\left\{A_{k}\right\}$ in $\mathcal{K}(M)$ such that $y_{k} \in \Gamma_{A_{k}}$ and $A_{k} \in V_{n_{k}}$.

Since $y_{0} \in B_{n_{1}}$ there exists $A_{1} \in V_{n_{1}}$ such that $U_{1} \cap \Gamma_{A_{1}} \neq \varnothing$; choose $y_{1} \in$ $U_{1} \cap \Gamma_{A_{1}}$. Now $y_{0} \neq y_{1}$, so pick a neighborhood $U_{2} \subseteq U_{1}$ of $y_{0}$ in $K$ such that $y_{1} \notin U_{2}$. Since $y_{0} \in B_{n_{2}}$ there exists $A_{2} \in V_{n_{2}}$ such that $U_{2} \cap \Gamma_{A_{2}} \neq \varnothing$; choose $y_{2} \in U_{2} \cap \Gamma_{A_{2}}$ and proceed in the same way.

It should be noted that the sequence $\left\{V_{n_{k}}: k \in \omega\right\}$ is a neighborhood base for $A$ in $\mathcal{K}(M)$ and since $A_{k} \in V_{n_{k}}, A_{k} \rightarrow A$ in $\mathcal{K}(M)$. Thus $\left\{A_{k}: k \in \omega\right\} \cup\{A\}$ is compact in $\mathcal{K}(M)$ and so $B=\bigcup_{k \in \omega} A_{k} \cup A$ is compact in $M$ (see $\S 0$ ). Now we have

$$
\left\{y_{k}: k<\omega\right\} \subseteq \bigcup_{k<\omega} \Gamma_{A_{k}} \subseteq \Gamma_{B}
$$

and since $\Gamma_{B} \cup\{\infty\}$ is compact in $F^{\infty}(M), y_{k} \rightarrow \infty$. This is a contradiction because $\left\{y_{k}: n<\omega\right\} \subseteq \bar{U}_{1}$ and $\infty \notin \bar{U}_{1}$.

In the case where $M$ is complete, there is $f: \Sigma \rightarrow K(M)$ continuous, onto (see $\S 0)$. We set $M_{\sigma}=\bigcup\{f(\rho): \rho \leq \sigma\}, \sigma \in \Sigma$. We observe that $M_{\sigma}$ is compact, $M_{\sigma} \subseteq M_{\rho}$ if $\sigma \leq \rho$ (see $\S 0$ ), and that if $K$ is compact in $M$ then $K \subseteq M_{\sigma}$ for some $\sigma \in \Sigma$. Thus for every $A \subseteq \Gamma$ we have: $\left|A \cap \Gamma_{K}\right|<\omega$ for every $K \in \mathcal{K}(M)$ if and only if $\left|A \cap \Gamma_{M_{\sigma}}\right|<\omega$ for every $\sigma \in \Sigma$. 
Now we set $\Gamma_{s}=\operatorname{cl}_{K}\left(\bigcup_{s<\sigma} \Gamma_{M_{\sigma}}\right)$ for every $s \in S$ and using the above remark we prove similarly that $F^{\infty}(M)=\bigcup_{\sigma \in \Sigma} \bigcap_{n=1}^{\infty} \Gamma_{\sigma \mid n}$, that is $F^{\infty}(M)$ is $K$-analytic.

REMARK 1.4.1. If $\left|\Gamma_{K}\right|<\omega$ for all $K \in \mathcal{K}(M)$ then $\Gamma$ is at most countable. Indeed, $\infty$ is an isolated point of $F^{\infty}(M)$, so $F^{\infty}(M)$ is a countably determined (and hence Lindelöf) discrete space, and thus the conclusion follows.

LEMMA 1.5. If $X$ is countably determined (resp. $K$-analytic) then $D^{\infty}(X)$ is countably determined (resp. $K$-analytic).

Proof. Assume that $X$ is countably determined and let $\phi: M \rightarrow \mathcal{K}(X)$ be usco and onto (see Proposition 0.1) where $M$ is a separable metric space. Consider the space $F^{\infty}(M)$ of Definition 1.3, where $\Gamma_{K}=\phi(K), K \in \mathcal{K}(M)$. Then $X=$ $\bigcup\left\{\Gamma_{K}: K \in \mathcal{K}(M)\right\}$ and it is easy to see that the "identity" $I: F^{\infty}(M) \rightarrow D^{\infty}(X)$ is continuous. Thus $D^{\infty}(X)$ is countably determined.

The $K$-analytic case is similar (setting $M=\Sigma$ ).

LEMMA 1.6. For every space $X$, there exists a linear isometry $T: C_{1}(X) \rightarrow$ $C\left(D^{\infty}(X)\right)$ that is continuous for the topology of pointwise convergence.

Proof. We define $T: C_{1}(X) \rightarrow C\left(D^{\infty}(X)\right)$ by

$$
T(f)(t)=\left\{\begin{array}{ll}
f(t), & \text { if } t \in X, \\
0, & \text { if } t=\infty,
\end{array} \quad \text { for every } f \in C_{1}(X) .\right.
$$

It is easy to see that $T$ has the desired properties.

PROOF OF THEOREM 1.2. Talagrand proved that every pointwise compact subset of $C(Y)$, where $Y$ is countably determined (resp. $K$-analytic), is Gulko compact (resp. Talagrand compact) (Theorem 3.7 of $[\mathbf{2 1}]$ ). Thus the proof follows immediately from Lemmas 1.4-1.6.

As consequences of Theorem 1.2 we have

Corollary 1.7. Let $\Gamma=\bigcup\left\{\Gamma_{K}: K \in \mathcal{K}(M)\right\}$ be as in Definition 1.3(i). If $\Omega$ is a compact subset of $\mathbf{R}^{\Gamma}$ such that for each $K \in \mathcal{K}(M), \Omega \mid \Gamma_{K} \subseteq C_{0}\left(\Gamma_{K}\right)$ then

(a) $\Omega \subseteq \Sigma\left(\mathbf{R}^{\Gamma}\right)$ (= the $\Sigma$-product of the real line),

(b) $\Omega$ is Gulko compact, and

(c) if $M=\Sigma$, then $\Omega$ is Talagrand compact.

Proof. We define $H: \Omega \rightarrow C_{1}\left(F^{\infty}(M)\right)$ by

$$
H(f)(t)= \begin{cases}f(t), & \text { if } t \in \Gamma \\ 0, & \text { if } t=\infty\end{cases}
$$

It is easy to see that $H$ is continuous when $C_{1}\left(F^{\infty}(M)\right)$ is endowed with the topology of pointwise convergence. Thus the result follows from Lemma 1.4 and Theorem 1.2 (see also Definition 1.1(d)).

COROLLARY 1.8. Let $\Omega$ be a compact space, $M$ a separable metric space and $\left\{L_{K}: K \in \mathcal{K}(M)\right\}$ a family of weakly compact subsets of $C(\Omega)$ such that

(a) for every $K \in \mathcal{K}(M)$ the only possible weak limit point of $L_{K}$ is $0 \in C(\Omega)$,

(b) if $K_{1}, K_{2} \in \mathcal{K}(M)$ with $K_{1} \subseteq K_{2}$ then $L_{K_{1}} \subseteq L_{K_{2}}$ and

(c) the set $L=\bigcup\left\{L_{K}: K \in \mathcal{K}(M)\right\}$ separates the points of $\Omega$.

Then $\Omega$ is a Gulko compact space; especially if $M=\Sigma, \Omega$ is a Talagrand compact.

Proof. The space $F^{\infty}(M)=\Gamma \cup\{\infty\}$ (Definition 1.3, where $\Gamma_{K}=L_{K} \backslash\{0\}$ ) is countably determined (Lemma 1.4). We define $f: F^{\infty}(M) \rightarrow L \cup\{0\}$ by $f(x)=x$ 
for $x \in \Gamma$, and $f(\infty)=0$. Then $f$ is continuous when the space $L \cup\{0\}$ has the topology of pointwise convergence, so $L \cup\{0\}$ is countably determined. The desired conclusion now follows from Theorem 3.4 of $[\mathbf{2 1}]$.

2. The main theorem. The content of this section is the proof of Theorem 2.5 (see Introduction); its consequences are examined in the next two sections.

We shall denote by $K$ a compact Hausdorff space. The topological concepts involved (closure, density, continuity, etc.) in $C(K)$ are always considered in the pointwise topology of $C(K)$. We shall refer frequently to the fundamental paper of Gulko $[\mathbf{9}]$, to the survey paper of Negrepontis $[\mathbf{1 6}, \S 6]$, and also to the basic paper of Talagrand [21].

Definition. Let $F \subseteq C(K), F$ separates points of $K, M \subseteq K, L \subseteq F$. We say that the pair $(M, L)$ is $F$-conjugate if

(a) for all $x \in K$, there is $p(x) \in M$, such that $f(x)=f(p(x))$ for all $f \in L$, and

(b) for all $f \in F$, there is $q(f) \in L$, such that $f(x)=(q(f))(x)$ for all $x \in M$.

LEMMA 2.1. Let $F \subseteq C(K), F$ separates points of $K, M \subseteq K, L \subseteq F$, with $(M, L) F$-conjugate. Then,

(a) $p(x), q(f)$ are unique for all $x \in K, f \in F$;

(b) the mappings $p: K \rightarrow M, q: F \rightarrow L$ defined by (a) are continuous retractions (so $M, L$ are closed subsets of $K, F$, respectively); and

(c) $p^{*} \mid F=q\left(\right.$ where $\left.p^{*}: C(K) \rightarrow C(K): p^{*}(f)=(f \mid M) \circ p\right)$.

Definition. Let $F \subseteq C(K), M \subseteq K, L \subseteq F$. We say that the pair $(M, L)$ is $F$-preconjugate if

(a) $\{x \mid L: x \in M\}$ is dense in $\{x \mid L: x \in K\}$ (in the pointwise topology of $L \subseteq$ $C(K))$, and

(b) $\{f \mid M: f \in L\}$ is dense in $\{f \mid M: f \in F\}$ (in the pointwise topology of $L \subseteq$ $F \subseteq C(K))$.

Here we use the notation: $x \mid L: L \rightarrow \mathbf{R}$ to mean $(x \mid L)(f)=f(x)$ for $f \in L$.

REMARK. The above two Definitions and Lemma 2.1 are due to Gulko [9] (see also [16, Definitions 6.11, 6.13 and Lemma 6.12]). We include them here, because they are useful in the sequel.

Lemmas 2.2 and 2.4 are fundamental for the proof of Theorem 2.5 and improve corresponding lemmas of Gulko [9] (see [16, Lemmas 6.19, 6.22]). First we need some preliminaries.

Let $K$ be a Gulko compact and $L$ a countably determined subspace of the closed unit ball of $C(K)$ for the pointwise topology which separates the points of $K[\mathbf{2 1}$, Theorem 3.4]. It is clear that $L$ is a countably determined subspace of the compact space $[-1,1]^{K}$ and so there are a nonempty subset $\Sigma^{\prime}$ of $\Sigma$ and a family $\left\{L_{s}: s \in S\right\}$ of compact subsets of $[-1,1]^{K}$ with $L_{\varnothing}=[-1,1]^{K}$ such that:

(a) for every $s_{1}, s_{2} \in S$ with $s_{1}<s_{2}, L_{s_{2}} \subseteq L_{s_{1}}$,

(b) for every $\sigma \in \Sigma^{\prime}, L_{\sigma}=\bigcap_{n<\omega} L_{\sigma \mid n} \neq \varnothing$ and

(c) $L=\bigcup_{\sigma \in \Sigma^{\prime}} \bigcap_{n<\omega} L_{\sigma \mid n}$.

Since the map $F: \Sigma^{\prime} \rightarrow \mathcal{K}(L): \sigma \in \Sigma^{\prime} \rightarrow L_{\sigma} \subseteq L$ is usco [21, Proposition 1.1] if $A$ is a compact subset of $\Sigma^{\prime}$, the set $L_{A}=\bigcup\left\{L_{\sigma}: \sigma \in A\right\}$ is a pointwise compact subset of $L$. 
For every $A \in \mathcal{K}\left(\Sigma^{\prime}\right)$ we define in the space $K$ an equivalence relation $R_{A}$ as follows:

$$
x R_{A} y \quad \text { iff } \quad f(x)=f(y) \text { for all } f \in L_{A} .
$$

Let $K_{A}=K / R_{A}$ be the quotient space and $\pi_{A}: K \rightarrow K_{A}$ the natural map. We endow the space $K_{A}$ with the quotient topology, that is, $U \subseteq K_{A}$ is open if and only if $\pi_{A}^{-1}(U)$ is open in $K$, and we notice that $\pi_{A}$ becomes a continuous open map and $K_{A}$ a compact Hausdorff space. We also notice that $K_{A}$ is an Eberlein compact, since $L_{A}$ is a pointwise compact subset of $C(K)$ which separates points of $K_{A}$.

Part (a) of the following lemma is due to Gulko [9] (see also [16, Lemma 6.19]). We include the proof because it is needed for parts (b) and (c). If $x \in K$ and $A \in \mathcal{K}\left(\Sigma^{\prime}\right),[x]^{A}$ will denote the equivalence class of $x$ with respect to $R_{A}$.

LEMMA 2.2. Let $M \subseteq K, N \subseteq \bigcup_{s \in S}\left(L_{s} \cap L\right)$ such that $\left(M, N \cap L_{s} \cap L\right)$ is $\left(L_{s} \cap L\right)$-preconjugate for $s \in S$. Set $P=\operatorname{cl}_{K} M, Q=\operatorname{cl}_{L} N \subseteq L$ and for $A \in \mathcal{K}\left(\Sigma^{\prime}\right), P_{A}=\pi_{A}(P) \subseteq K_{A}, Q_{A}=Q \cap L_{A} \subseteq L_{A}$. Then

(a) $(P, Q)$ is L-conjugate.

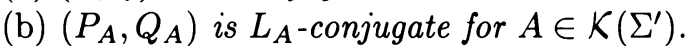

(c) If $(p, q)$ and $\left(p^{A}, q^{A}\right)$ denote the retractions corresponding to the conjugate pairs $(P, Q)$ and $\left(P_{A}, Q_{A}\right)$, respectively (see Lemma 2.1), then $p^{A}\left([x]^{A}\right)=[p(x)]^{A}$ for $[x]^{A} \in K_{A}$ and $q^{A}(f)=q(f) \circ \pi_{A}=q\left(f \circ \pi_{A}\right)$ for $f \in L_{A}$.

Proof. (a) Let $f \in L$. Then there exists $\sigma \in \Sigma^{\prime}$ such that $f \in L_{\sigma}=$ $\bigcap_{n<\omega} L_{\sigma \mid n} \subseteq L$. We set for $n<\omega$,

$$
V(f, \sigma \mid n)=\left\{g \in \operatorname{cl}_{[-1,1]^{K}}\left(L_{\sigma \mid n} \cap Q\right): g|M=f| M\right\} \subseteq L_{\sigma \mid n} \cap \mathrm{cl}_{[-1,1]^{K}} Q .
$$

Since $\left(M, N \cap L_{\sigma \mid n} \cap L\right)$ is $\left(L_{\sigma \mid n} \cap L\right)$-preconjugate, and $f \in L_{\sigma \mid n} \cap L$, there is a net $\left(g_{i}\right)_{i \in I} \subseteq N \cap L_{\sigma \mid n} \cap L \subseteq L_{\sigma \mid n} \cap Q$ such that $\lim _{i \in I}\left(g_{i} \mid M\right)=f \mid M$. If $g$ is a cluster point of this net, then it follows easily that $g \in V(f, \sigma \mid n)$, so $V(f, \sigma \mid n)$ is a nonempty compact subset of $[-1,1]^{K}$. We notice that if $n>m$ then $V(f, \sigma \mid n) \subseteq V(f, \sigma \mid m)$. Therefore

$$
\begin{aligned}
V_{f} & =\bigcap_{n<\omega} V(f, \sigma \mid n) \subseteq \bigcap_{n<\omega}\left(L_{\sigma \mid n} \cap \mathrm{cl}_{[-1,1]^{K}} Q\right) \\
& =L_{\sigma} \cap \operatorname{cl}_{[-1,1]^{K}} Q \subseteq L \cap \mathrm{cl}_{[-1,1]^{K}} Q=Q .
\end{aligned}
$$

So we have that $V_{f}$ is a nonempty compact subset of $Q$, and thus there is $g \in V_{f} \subseteq Q$ such that $g|M=f| M$. Since $f, g$ are continuous, it follows that $f|\bar{M}=g| \bar{M}$, that is, condition (b) of the conjugate pair.

Since $\left(M, N \cap L_{\varnothing} \cap L\right)(=(M, N))$ is $L_{\varnothing} \cap L(=L)$-preconjugate, it follows that $\{x \mid Q: x \in P\}=\{x \mid Q: x \in K\}[\mathbf{1 6}$, Lemma 6.17], that is, condition (a) of the conjugate pair. Therefore $(P, Q)$ is $L$-conjugate pair.

(b) Let $A \in \mathcal{K}\left(\Sigma^{\prime}\right)$ and $f \in L_{A} \subseteq L$. Then there exists $\sigma \in A$ such that $f \in L_{\sigma} \subseteq L_{A}$. as in the proof of the claim (a), there exists $g \in V_{f} \subseteq L_{\sigma} \cap Q \subseteq L_{A} \cap Q$ such that $f(x)=g(x)$ for all $x \in P($ see $(*))$.

Since $f, g \in L_{\sigma} \subseteq L_{A}$, it follows by the definition of the space $K_{A}$, that $f \circ \pi_{A}, g \circ$ $\pi_{A} \in L_{A} \subseteq C\left(K_{A}\right)$ and $f \circ \pi_{A}(x)=g \circ \pi_{A}(x)$ for every $x \in P$, so $f\left([x]^{A}\right)=g\left([x]^{A}\right)$ for every $[x]^{A} \in P_{A}$. 
Let $[x]^{A} \in K_{A}$ and $f_{0} \in Q_{A}\left(=L_{A} \cap Q \subseteq Q\right)$. There is $y \in P$ such that $x|Q=y| Q$, so $[y]^{A} \in P_{A}$ and $f_{0}(x)=f_{0}(y)$. It follows that $f_{0}\left([x]^{A}\right)=f_{0}\left([y]^{A}\right)$.

Now since $f_{0} \in L_{A}$ we have that $f_{0} \circ \pi_{A} \in L_{A} \subseteq C\left(K_{A}\right)$. The proof of (b) is complete.

(c) Follows immediately from (a) and (b).

LEMMA 2.3. If $K$ is a Gulko compact space, and $L$ a subset of the unit ball of $C(K)$ that separates the points of $K$ in the pointwise topology, then $W(K)=$ $d(K)=d(L)$.

ProOF. We refer to Talagrand [21, Theorem 6.2 and Proposition 6.3].

LeMma 2.4. Assume that $W(K)=\tau \geq \omega^{+}$and let $\left\{x_{\xi}: \omega \leq \xi<\tau\right\}$ and $\left\{f_{\xi}: \omega \leq \xi<\tau\right\}$ be dense subsets of $K$ and $L$, respectively. (We recall that $L$ is considered with the pointwise topology.) Then

(a) There exists a family $\left\{\left(P_{\xi}, Q_{\xi}\right): \omega \leq \xi<\tau\right\}$ such that

(1) $\left(P_{\xi}, Q_{\xi}\right)$ is L-conjugate, $\omega \leq \xi<\tau$.

(2) $P_{\xi} \subseteq P_{\zeta}$ and $Q_{\xi} \subseteq Q_{\zeta}, \omega \leq \xi<\varsigma<\tau$.

(3) If $\xi$ is a limit ordinal, $\omega<\xi<\tau$, then $P_{\xi}=\mathrm{cl}_{K}\left(\bigcup_{n<\xi} P_{n}\right), Q_{\xi}=$ $\operatorname{cl}_{L}\left(\bigcup_{n<\xi} Q_{n}\right)$.

(4) $x_{\xi} \in P_{\xi+1}$ and $f_{\xi} \in Q_{\xi+1}$, for $\omega \leq \xi<\tau$.

(5) $W\left(P_{\xi}\right)=d\left(P_{\xi}\right)=d\left(Q_{\xi}\right) \leq|\xi|$ for $\omega \leq \xi<\tau$.

(6) $\lim _{n<\xi} p_{n}(x)=p_{\xi}(x)$ for $x \in K$ and $\xi$ limit ordinal, $\omega<\xi<\tau$, where $p_{\xi}$ is the retraction from $K$ onto $P_{\xi}$ of Gulko's Lemma 2.1 .

(7) For every $x \in K$ the set $\left\{\xi: \omega \leq \xi<\tau\right.$ and $\left.p_{\xi}(x) \neq p_{\xi+1}(x)\right\}$ is countable.

(b) For every $A \in \mathcal{K}\left(\Sigma^{\prime}\right)$ there is a family $\left\{\left(P_{\xi}^{A}, Q_{\xi}^{A}\right): \omega \leq \xi<\tau\right\}$ such that

(1) $\left(P_{\xi}^{A}, Q_{\xi}^{A}\right)$ is $L_{A}$-conjugate, where $P_{\xi}^{A}=\pi_{A}\left(P_{\xi}\right)$ and $Q_{\xi}^{A}=Q_{\xi} \cap L_{A}$ for $\omega \leq \xi<\tau$.

(2) $P_{\xi}^{A} \subseteq P_{\varsigma}^{A}$ and $Q_{\xi}^{A} \subseteq Q_{\zeta}^{A}$ for $\omega \leq \xi<\varsigma<\tau$.

(3) If $\xi$ is a limit ordinal,' $\omega<\xi<\tau$, then $P_{\xi}^{A}=\operatorname{cl}_{K_{A}}\left(\bigcup_{n<\xi} P_{n}^{A}\right)$ and $Q_{\xi}^{A}=$ $\mathrm{cl}_{L_{A}}\left(\bigcup_{n<\xi} Q_{n}^{A}\right)$.

(4) $\pi_{A}\left(x_{\xi}\right) \in P_{\xi+1}^{A}$ and $f_{\xi} \circ \pi_{A} \in Q_{\xi+1}^{A}$ for $\omega \leq \xi<\tau$.

(5) $W\left(P_{\xi}^{A}\right)=W\left(Q_{\xi}^{A}\right) \leq|\xi|$ for $\omega \leq \xi<\tau$.

(6) If $\xi$ is a limit ordinal with $\omega \leq \xi<\tau$ then $\lim _{n<\xi} p_{n}^{A}\left([x]^{A}\right)=p_{\xi}^{A}\left([x]^{A}\right)$, for all $[x]^{A} \in K_{A}$, where $p_{\xi}^{A}$ is the retraction from $K_{A}$ onto $P_{\xi}^{A}$ given by $p_{\xi}^{A}\left([x]^{A}\right)=$ $\left[p_{\xi}(x)\right]^{A}$ (see Lemma 2.2(c)).

(c) (1) For every ordinal $\xi, \omega \leq \xi<\tau, Q_{\xi}=\bigcup\left\{Q_{\xi}^{A}: A \in \mathcal{K}\left(\Sigma^{\prime}\right)\right\}$ and for every $A, B \in \mathcal{K}\left(\Sigma^{\prime}\right)$ with $A \subseteq B, Q_{\xi}^{A} \subseteq Q_{\xi}^{B}$.

(2) For every $A \in \mathcal{K}\left(\Sigma^{\prime}\right)$ the retractions corresponding to the conjugate pair $\left(P_{\xi}^{A}, Q_{\xi}^{A}\right)$ are the following:

$$
\begin{aligned}
& p_{\xi}^{A}: K_{A} \rightarrow P_{\xi}^{A} \subseteq K_{A}:[x]^{A} \rightarrow\left[p_{\xi}(x)\right]^{A}, \\
& q_{\xi}^{A}: L_{A} \rightarrow Q_{\xi}^{A} \subseteq L_{A}: f \rightarrow q_{\xi}(f) \circ \pi_{A}=q_{\xi}\left(f \circ \pi_{A}\right) .
\end{aligned}
$$

Proof. The main tool of the proof of (a) is Lemma 2.2(a) and is due to Gulko [9] (see [16, Lemma 6.22]). (b) and (c) follow easily from (a) and Lemma 2.2(b) and (c). 
ProOF OF THEOREM 2.5. We distinguish two cases.

Case 1. The set $L$ is bounded.

We set $K=B_{F^{*}}$ with the weak* topology, where $F$ is the closed linear span of $L$ in $E$, so $F$ is a WCD Banach space and $K$ a Gulko compact space by Theorem 3.6 of [21]. Since $F$ is naturally embedded in $C(K), L$ is a countably determined subset of $C(K)$ for the pointwise topology and separates points of $K$. It is clear that we can assume without loss of generality that $L$ is contained in the closed unit ball of $C(K)$.

Claim. There exists a family $\left\{T_{A}: A \in \mathcal{K}\left(\Sigma^{\prime}\right)\right\}$ of bounded linear one-to-one operators $T_{A}: C\left(L_{A}\right) \rightarrow C_{0}\left(A \times\{0,1\}^{\alpha}\right)$, where $L_{A}$ is as in Lemma 2.2, such that:

(a) if $A \in \mathcal{K}\left(\Sigma^{\prime}\right)$ then $\left\|T_{A}\right\| \leq 1$ and $T_{A}$ is pointwise to pointwise continuous; and

(b) if $A, B \in \mathcal{K}\left(\Sigma^{\prime}\right)$ with $A \subseteq B$, then $T_{B}(f) \mid A \times\{0,1\}^{\alpha}=T_{A}(f)$ for every $f \in C(L)$.

The claim is proved inductively. So suppose that the claim holds when $L$ is such that $d(L)<\kappa$. (The case $d(L)=\omega$ is trivial: for every $A \in \mathcal{K}\left(\Sigma^{\prime}\right)$ we set $T_{A}=T: C(L) \rightarrow C_{0}(\omega) \subseteq C_{0}\left(\{0,1\}^{\omega}\right)$ such that $T(f)(n)=f\left(d_{n}\right) / n, n<\omega$, where $\left\{d_{n}: n<\omega\right\}$ is a dense subset of $L$.) Consider an $L$ with $d(L)=\kappa$. Let $\left\{\left(P_{\xi}, Q_{\xi}\right): \omega \leq \xi<\kappa\right\}$ and $\left\{\left(P_{\xi}^{A}, Q_{\xi}^{A}\right): \omega \leq \xi<\kappa, A \in \mathcal{K}\left(\Sigma^{\prime}\right)\right\}$ be as in Lemma 2.4. Since $d\left(Q_{\xi}\right) \leq|\xi|<\kappa$ by the induction hypothesis, there exists a family of operators, $T_{A}^{\xi}: C\left(Q_{\xi}^{A}\right) \rightarrow C_{0}\left(A \times\{0,1\}^{\alpha_{\xi}}\right), A \in \mathcal{K}\left(\Sigma^{\prime}\right)$, as in the claim. As $\alpha_{\xi} \leq \alpha_{\kappa}$, the range of each $T_{A}^{\xi}$ is included in $C_{0}\left(A \times\{0,1\}^{\alpha_{\kappa}}\right)$. Thus, if $t: \kappa \ni$ $\xi \rightarrow t_{\xi} \in\{0,1\}^{\alpha_{\kappa}}$ is a one-to-one mapping, the range of each $T_{A}^{\xi}$ is considered as a subset of $C_{0}\left(\left\{t_{\xi}\right\} \times A \times\{0,1\}^{\alpha_{\kappa}}\right)$.

We set $Y_{A}=\{0,1\}^{\alpha_{\kappa}} \times\left(A \times\{0,1\}^{\alpha_{\kappa}}\right)$ for $A \in \mathcal{K}\left(\Sigma^{\prime}\right)$ and $Y=\{0,1\}^{\alpha_{\kappa}} \times\left(\Sigma^{\prime} \times\right.$ $\left.\{0,1\}^{\alpha_{\kappa}}\right)$; it is obvious that if $A \in \mathcal{K}\left(\Sigma^{\prime}\right)$ the spaces $Y$ and $Y_{A}$ are homeomorphic to $\Sigma^{\prime} \times\{0,1\}^{\alpha_{\kappa}}$ and $A \times\{0,1\}^{\alpha_{\kappa}}$, respectively. For $A \in \mathcal{K}\left(\Sigma^{\prime}\right)$ we define

$$
T_{A}: C\left(L_{A}\right) \rightarrow C_{0}\left(\bigcup_{\omega \leq \xi<\kappa}\left[\left\{t_{\xi}\right\} \times A \times\{0,1\}^{a_{\kappa}}\right]\right) \subseteq C_{0}\left(Y_{A}\right)
$$

with $T_{A}(f)(n)=T_{\omega}\left(q_{\omega}^{A}(f)\right)(n), n<\omega$,

$$
\begin{gathered}
T_{A}(f)(\gamma)=\frac{1}{2}\left[T_{A}^{\xi+1}\left(q_{\xi+1}^{A}(f)-q_{\xi}^{A}(f)\right)(\gamma)\right], \quad \gamma \in\left\{t_{\xi+1}\right\} \times A \times\{0,1\}^{\alpha_{\kappa}}, \omega \leq \xi<\kappa, \\
T_{A}(f)(\gamma)=0, \quad \gamma \in Y_{A} \backslash \bigcup_{\omega \leq \xi<\kappa}\left[\left\{t_{\xi}\right\} \times A \times\{0,1\}^{\alpha_{\kappa}}\right] .
\end{gathered}
$$

From Lemma 2.4 and the induction hypothesis it follows that the family $\left\{T_{A}: A \in\right.$ $\left.\mathcal{K}\left(\Sigma^{\prime}\right)\right\}$ satisfies the claim.

Now we set

$$
T: C(L) \rightarrow C_{1}\left(\Sigma^{\prime} \times\{0,1\}^{\alpha_{\kappa}}\right)
$$

such that $T(f)=\left[T_{A}(f)\right]_{A \in \mathcal{K}\left(\Sigma^{\prime}\right)}$, for every $f \in C(L)$. It is clear from the claim that the operator $T$ has the desired properties.

Case 2. The set $L$ is unbounded.

For $n<\omega$ set $L_{n}=L \cap B_{n}$ where $B_{n}$ is the $n$-ball of $E$. Then, by case 1 , for every $L_{n}$ there exists an operator

$$
T_{n}: C\left(L_{n}\right) \rightarrow C_{1}\left(\Sigma_{n} \times\{0,1\}^{\alpha_{n}}\right)
$$


as in the statement of the theorem. Since $d\left(L_{n}\right) \leq d(L) \leq 2^{\alpha}$ for $n<\omega$, we consider the range of $T_{n}$ as a subset of $C_{1}\left(\{n\} \times \Sigma_{n} \times\{0,1\}^{\alpha}\right)$.

Now we set $T: C(L) \rightarrow C_{1}\left(\Sigma^{\prime} \times\{0,1\}^{\alpha}\right)$, where $\Sigma^{\prime}=\left(\bigcup_{n<\omega}\{n\} \times \Sigma_{n}\right)$, such that $T(f)=\left(n^{-1} T_{n}\left(f \mid L_{n}\right)\right)_{n<\omega}$ for every $f \in C(L)$. It is easily seen that $T$ satisfies the claim.

In the case where $L$ is weakly $K$-analytic it follows immediately from Lemma 2.2 and the comments before it that $\Sigma^{\prime}$ can be replaced by $\Sigma$.

The proof of the theorem is thus complete.

3. Topological consequences. We now come to some consequences of Theorems 2.5 and 1.2 .

TheOREM 3.1 (REPRESENTATION OF Gulko COMPACT SPACES). For $a$ compact space $\Omega$, the following are equivalent:

(a) $\Omega$ is a Gulko compact space;

(b) $\Omega$ is homeomorphically embedded in $C_{1}\left(\Sigma^{\prime} \times\{0,1\}^{\alpha}\right)$ endowed with the pointwise topology, for some $\Sigma^{\prime} \subseteq \Sigma$ and some infinite cardinal $\alpha$.

(c) $\Omega$ is homeomorphically embedded in $C_{1}(Y)$ endowed with the pointwise topology, for some countably determined space $Y$.

Proof. (a) $\Rightarrow(\mathrm{b})$ We have that $C(\Omega)$ is WCD. By Theorem 2.5 there is a one-to-one operator $T: C(C(\Omega)) \rightarrow C_{1}\left(\Sigma^{\prime} \times\{0,1\}^{\alpha}\right)$ that is pointwise to pointwise continuous. Since $\Omega$ is homeomorphic to a pointwise compact subset of $C(C(\Omega))$, (b) follows.

(b) $\Rightarrow$ (c) is obvious and (c) $\Rightarrow$ (a) follows from Theorem 1.2.

Analogously, we have the following theorem for Talagrand compact spaces.

THEOREM 3.2. For a compact space $\Omega$ the following are equivalent:

(a) $\Omega$ is a Talagrand compact space;

(b) $\Omega$ is homeomorphically embedded in $C_{1}\left(\Sigma \times\{0,1\}^{\alpha}\right)$ endowed with the pointwise topology, for some infinite cardinal $\alpha$.

(c) $\Omega$ is homeomorphically embedded in $C_{1}(Y)$ endowed with pointwise topology, for some $K$-analytic space $Y$.

The following theorem is similar to Rosenthal's characterization of Eberlein compact spaces $[\mathbf{1 9}]$. Before we formulate it, recall that, if $\mathcal{F}$ is a family of subsets of a set $X$ then we say that

(a) $\mathcal{F}$ separates the points $X$ if for every $x, y \in X$ with $x \neq y$ there exists $A \in \mathcal{F}: \chi_{A}(x) \neq \chi_{A}(y)$.

(b) $\mathcal{F}$ is point-finite (resp. point-countable) if every point of $X$ belongs to at most a finite (resp. a countable) number of members of $\mathcal{F}$.

THEOREM 3.3 (TOPOLOGICAL CHARACTERIZATION OF GULKO COMPACT SPACES). For a compact space $\Omega$, the following are equivalent:

(a) $\Omega$ is a Gulko compact space.

(b) There are a nonempty subset $\Sigma^{\prime}$ of $\Sigma$, a point-countable family $₹$ of open $F_{\sigma}$ subsets of $\Omega$, which separates points of $\Omega$, and a family $\left\{\exists_{A}: A \in \mathcal{K}\left(\Sigma^{\prime}\right)\right\}$ of subsets of $₹$ such that

(i) $\mathcal{F}=\bigcup\left\{\mathcal{F}_{A}: A \in \mathcal{K}\left(\Sigma^{\prime}\right)\right\}$;

(ii) if $A, B \in \mathcal{K}\left(\Sigma^{\prime}\right), A \subseteq B$ then $\mathcal{F}_{A} \subseteq \mathcal{F}_{B}$; and

(iii) if $A \in \mathcal{K}\left(\Sigma^{\prime}\right)$, then $\xi_{A}$ is a point-finite family. 
Proof. (b) $\Rightarrow$ (a) Let $A \in \mathcal{K}\left(\Sigma^{\prime}\right)$ and $\alpha \in \mathcal{F}_{A}$. Since $\alpha$ is an open $F_{\sigma}$ subset of $\Omega$ there exists a continuous function $f_{A}^{\alpha}: \Omega \rightarrow[0,1]$ such that $\alpha=\left\{x \in K: f_{A}^{\alpha}(x) \neq 0\right\}$. Let $L_{A}=\left\{f_{A}^{\alpha}: \alpha \in \mathcal{F}_{A}\right\} \cup\{0\}$. Using the fact that $\mathcal{F}_{A}$ is a point-finite family, it is easily seen that $L_{A}$ is a weakly compact subset of $C(\Omega)$, whose only weak limit point is $0 \in C(\Omega)$. Moreover, if $A, B \in \mathcal{K}\left(\Sigma^{\prime}\right)$ and $A \subseteq B$ then from condition (ii), $L_{A} \subseteq L_{B}$.

Set $L=\bigcup\left\{L_{A}: A \in \mathcal{K}\left(\Sigma^{\prime}\right)\right\}$. The conclusion now follows from Corollary 1.8, since $\mathcal{F}$ separates the points of $\Omega$.

(a) $\Rightarrow$ (b) We consider the family of sets $\mathcal{F}=\left\{\Gamma_{A}=A \times\{0,1\}^{\alpha}: A \in \mathcal{K}\left(\Sigma^{\prime}\right)\right\}$ of assertion (b) of Theorem 3.1. For every $A \in \mathcal{K}\left(\Sigma^{\prime}\right)$ and $n<\omega$, let $\mathcal{F}_{A}^{n}$ be the family of sets of the form $U_{\gamma, j}^{(A, n)}=\left\{x:(j-2) / n<\pi_{\gamma}(x)<j / n\right\}$, where $3 \leq j \leq n+1$ or $-n+1 \leq j \leq-1, \gamma \in \Gamma_{A}$, and $\pi_{\gamma}: \Omega \rightarrow \mathbf{R}$ the projection at point $\gamma \in \Gamma_{A}$. It is clear that $\mathcal{F}_{A}^{n}$ consists of open $F_{\sigma}$ sets and

$$
\bigcup_{j} U_{\gamma, j}^{(A, n)}=\left\{x:\left|\pi_{\gamma}(x)\right|>\frac{1}{n}\right\} .
$$

The family $\mathcal{F}_{A}^{n}$ is point-finite. Indeed, suppose that there is some $k \in \Omega$ belonging to infinitely many members of $\mathcal{F}_{A}^{n}$. Then there exists $j$ and a sequence $\left(\gamma_{i}\right)_{i<\omega}$ of distinct points of $\Gamma_{A}$, such that $k \in \bigcap_{i=1}^{\infty} U_{\gamma_{i}}^{(A, n)}$. From (1) we have that $\left|\pi_{\gamma_{i}}(k)\right|>$ $1 / n$ for all $i<\omega$, which is absurd since $\left(\gamma_{i}\right)_{i<\omega} \subseteq \Gamma_{A}$ and by Theorem 3.1(b) and Definition $1.1 \pi_{\gamma_{i}}(k)=k\left(\gamma_{i}\right) \rightarrow 0$.

For every compact subset $A$ of $\omega \times \Sigma^{\prime}$, set $\mathcal{F}_{A}=\bigcup\left\{\mathcal{F}_{\pi_{2}(A)}^{k}: k \in \pi_{1}(A)\right\}$ where $\pi_{1}: \omega \times \Sigma^{\prime} \rightarrow \omega$ and $\pi_{2}: \omega \times \Sigma^{\prime} \rightarrow \Sigma^{\prime}$ are the projections onto the first and second coordinates. We notice that the family $\mathcal{F}_{A}$ is point-finite. Indeed, since $A$ compact, $\pi_{2}(A)$ compact and $\pi_{1}(A)$ is finite subset of $\omega$. Also, it is clear that if $A, B \in$ $\mathcal{K}\left(\omega \times \Sigma^{\prime}\right)$ and $A \subseteq B$ then $\mathcal{F}_{A} \subseteq \mathcal{F}_{B}$.

Set $\mathcal{F}=\bigcup\left\{\mathcal{F}_{A}: A \in \mathcal{K}\left(\omega \times \Sigma^{\prime}\right)\right\}$. As in the remark following Lemma 1.4, we see that $\mathcal{F}$ is point-countable. It remains to prove that the family $\mathcal{F}$ separates the points of $\Omega$. Let $x, y$ be distinct points of $\Omega$. Then there is $\gamma$ such that $\pi_{\gamma}(x) \neq \pi_{\gamma}(y)$, say $\pi_{\gamma}(x)<\pi_{\gamma}(y)$ and $\pi_{\gamma}(y)>0$. Then there is $n<\omega$ such that $\pi_{\gamma}(y)>1 / n$ and $\pi_{\gamma}(y)-\pi_{\gamma}(x)>2 / n$. Therefore for some $j$ with $3 \leq j \leq n+1$ and some $A \in \mathcal{K}\left(\Sigma^{\prime}\right), y \in U_{\gamma, j}^{(A, n)}$ and $x \notin U_{\gamma, j}^{(A, n)}$, where $U_{\gamma, j}^{(A, n)} \in \mathcal{F}_{A}^{n} \subseteq \mathcal{F}$.

REMARKS 3.3.1. G. Gruenhage [10], using Theorem 3.3, proved that every Gulko compact space contains a $G_{\delta}$ dense metrizable subset. This answers a question of Talagrand [21, 22] and implies a result of Argyros and Negrepontis [3].

If $\Omega$ is Gulko compact totally disconnected space, then there exists a family $\mathcal{F}^{\prime}$ of clopen sets which satisfies assertion (b) of the preceding theorem. Indeed, for every $\alpha \in \mathcal{F}$, we can choose an increasing sequence $\left(\alpha_{k}\right)_{k \in \omega}$ of open and closed subsets of $\Omega$ such that $\alpha=\bigcup_{k<\omega} \alpha_{k}$. Now, for $A \in \mathcal{K}\left(\Sigma^{\prime}\right)$ and for $n \in \omega$ set $\mathcal{F}_{(n, A)}=\left\{\alpha_{i}: i \leq\right.$ $\left.n, a \in \mathcal{F}_{A}\right\}$ and for $B \in \mathcal{K}\left(\omega \times \Sigma^{\prime}\right)$ set $\mathcal{F}_{B}=\bigcup\left\{\mathcal{F}_{\left(k, \pi_{2}(B)\right)}: k \in \pi_{1}(B)\right\}$. It is easily seen that the family $\mathcal{F}^{\prime}=\bigcup\left\{\mathcal{F}_{B}: B \in \mathcal{K}\left(\omega \times \Sigma^{\prime}\right)\right\}$ has the desired properties.

PROPOSITION 3.4. For a compact space $\Omega$ the following are equivalent:

(a) $\Omega$ is a totally disconnected Gulko compact space.

(b) $\Omega$ is homeomorphic to a pointwise compact subset of $C_{1}(Y)$ consisting of characteristic functions, for some space $Y$ of the form $F^{\infty}(M)$ (see Definition 1.3). 
(c) $\Omega$ is homeomorphic to a pointwise compact subset of $C_{1}(Y)$ consisting of characteristic functions, for some countably determined space $Y$.

Proof. (a) $\Rightarrow$ (b) Let $\mathcal{F}=\bigcup\left\{\mathcal{F}_{A}: A \in \mathcal{K}\left(\Sigma^{\prime}\right)\right\}$ be a family of open and closed subsets of $\Omega$ that satisfies (b) of Theorem 3.3 (see also the above remarks).

For $K \in \mathcal{K}\left(\Sigma^{\prime}\right)$ set $\Gamma_{K}=\mathcal{F}_{K}, M=\Sigma^{\prime}$ and $F=\left\{\Gamma_{K}: K \in \mathcal{K}\left(\Sigma^{\prime}\right)\right\}$. It is clear from the properties of $\mathcal{F}$ that the mapping $\Phi: x \in \Omega \rightarrow\left(X_{\alpha}(x)\right)_{\alpha \in \mathcal{F}} \in C_{1}\left(F^{\infty}(M)\right)$ is a homeomorphic embedding.

(b) $\Rightarrow$ (c) is obvious.

(c) $\Rightarrow$ (a) By Theorem 1.2, $\Omega$ is Gulko compact. Since $\Omega$ is embedded in $\{0,1\}^{Y}$, it is also totally disconnected.

REMARK 3.4.1. It is clear, as in Theorem 3.2, that the analogues of 3.3 and 3.4 hold for Talagrand compact spaces.

Using Theorem 3.1 we obtain a result connecting Gulko compact spaces with the complicated class of Rosenthal compact spaces. We recall that a compact space is said to be Rosenthal compact if it is homeomorphic to a pointwise compact subset of the space $B_{1}(M)$ of first Baire class real-valued functions on some Polish space $M$ (see [20 and 16, Definitions 1.10, 1.12]).

THEOREM 3.5. Every Gulko-compact space $\Omega$ of weight at most $2^{\omega}$ is Rosenthal-compact.

PROOF. According to Theorem 3.1, we have that there exists a homeomorphic embedding of $\Omega$ into a space of the form $C_{1}\left(\Sigma^{\prime} \times\{0,1\}^{\omega}\right)$ for some subset $\Sigma^{\prime}$ of $\Sigma$. Consider a completion $\hat{M}$ of the separable metric space $M=\Sigma^{\prime} \times\{0,1\}^{\omega}$. By the definition of Rosenthal-compact spaces it suffices to prove that the space $C_{1}(M)$ is included in the space $B_{1}(\hat{M})$.

For every $f \in C_{1}(M)$, we define $\hat{f}: \hat{M} \rightarrow \mathbf{R}$ by

$$
\hat{f}(x)= \begin{cases}0, & x \in \hat{M} \backslash M, \\ f(x), & x \in M\end{cases}
$$

For every $\varepsilon>0$, the set $\sigma_{\varepsilon}(f)=\{t \in M:|f(x)| \geq \varepsilon\}$ is by definition a closed and discrete subset of $M$, and so a countable $G_{\delta}$ subset of $\hat{M}$.

Now, it is easily seen that the inverse image under $\hat{f}$ of every open subset of $\mathbf{R}$ is $F_{\sigma}$ in $\hat{M}$, so $\hat{f}$ is first Baire class [16, Theorem 1.11] and thus $C_{1}(M) \subseteq B_{1}(\hat{M})$.

The following result is due to Professor S. Argyros, who permitted us to inlcude it in our paper.

THEOREM 3.6. Suppose that $K$ is a Talagrand (Gulko) compact set which is considered as a subset of the space $\Sigma[0,1]^{\Gamma}$ for some set $\Gamma$. Then there exists a family $\left\{\Gamma_{\sigma}, \sigma \in \Sigma\right\}\left(\left\{\Gamma_{\sigma}, \sigma \in \Sigma^{\prime}, \Sigma^{\prime} \subset \Sigma\right\}\right)$ such that for $\sigma \leq \sigma^{\prime}, \Gamma_{\sigma} \subset \Gamma_{\sigma^{\prime}}$ and $K \mid \Gamma_{\sigma}$ is a subset of $C_{0}\left(\Gamma_{\sigma}\right)$.

PROOF. We prove the result for Talagrand compact sets. The case of Gulko compact sets follows from similar arguments. Since $K$ is Talagrand compact $C(K)$ endowed with the weak topology is $K$-analytic hence there exists a family $\left\{L_{\sigma}: \sigma \in\right.$ $\Sigma$ \} of weakly compact sets of $C(K)$ ordered as the irrationals (i.e. $\sigma \leq \sigma^{\prime}, L_{\sigma} \subset L_{\sigma^{\prime}}$ ) and $\bigcup_{\sigma \in \Sigma} L_{\sigma}=C(K)$.

In the sequel, for $\gamma \in \Gamma, \pi_{\gamma}: \Sigma[0,1]^{\Gamma} \rightarrow[0,1]$ denotes the usual projection on the $\gamma$-coordinate. We also denote by $\Gamma_{\sigma}$ the set $\left\{\gamma \in \Gamma: \pi_{\gamma} \mid K \in L_{\sigma}\right\}$. 
Claim. For every subset $E$ of $\Gamma$ there exists a partition $\left\{E_{n}\right\}_{n<\omega}$ of $E$ so that the set $\left\{\pi_{\gamma} \mid K: \gamma \in \Gamma_{\sigma} \cap E_{n}\right\}$ is weakly discrete and has as a unique weak limit point the element $0 \in C(K)$.

Assume that the claim has been proved. Then we finish the proof of the theorem in the following way.

For $E=\Gamma$ we get a sequence $\left\{\Gamma_{n}\right\}_{n<\omega}$ satisfying the claim and set $\Delta_{n}=\bigcup_{k=1}^{n} \Gamma_{k}$ and $\Gamma_{(\sigma, n)}=\Gamma_{\sigma} \cap \Delta_{n}$. The set $\Sigma \times \mathbf{N}$ which is homeomorphic to $\Sigma$ and it is obvious that if $(\sigma, n) \leq\left(\sigma^{\prime}, m\right)$ then $\Gamma_{(\sigma, n)} \subset \Gamma_{\left(\sigma^{\prime}, m\right)}$.

Finally, let $x \in K$. Then $x \mid \Gamma_{(\sigma, n)} \in C_{0}\left(\Gamma_{(\sigma, n)}\right)$. Indeed, if this is not the case there exists an infinite sequence $\left\{\gamma_{K}\right\}_{K<\omega}$ in $\Gamma_{(\sigma, n)}$ with $\left|\pi_{\gamma_{K}}(x)\right|>\theta>0$, which contradicts our assumption that $\left\{\pi_{\gamma_{K}} \mid K\right\}_{K<\omega}$ converges weakly to zero.

PROOF OF THE CLAIM. We assume that $|\Gamma|=w(K)$ and we prove the claim using transfinite induction on the cardinality of the subsets $\Delta$ of $\Gamma$. It is clear that the claim is true for all $\Delta \subset \Gamma$ with $|\Delta|=\omega$.

Consider $\alpha<|\Gamma|$ and assume that for all $\beta<\alpha$ the inductive hypothesis is satisfied.

The following lemma is contained in [16, Lemma 6.25].

LEMMA. For a compact subset $K$ of $\Sigma\left([0,1]^{\Gamma}\right)$, there exists an increasing family $\Gamma_{1} \subseteq \Gamma_{2} \subseteq \cdots \subseteq \Gamma_{\xi} \subseteq \cdots \subseteq \Gamma_{\tau}=T$ (where $\left.|\Gamma|=\tau\right)$ with $\left|\Gamma_{\xi}\right|<\tau$ for $\xi<\tau$, and $\Gamma_{n}=\bigcup_{\xi<n} \Gamma_{\xi}$ for limit $n \leq \tau$, such that for all $\xi \leq \tau$ and $x \in K, x \mid \Gamma_{\xi} \in K$.

Consider a subset $\Delta$ of $\Gamma$ with $|\Delta|=\alpha>\omega$. From the above lemma there exists a "long" sequence $\left\{\Gamma_{\xi}\right\}_{\xi<\alpha}$ satisfying the conclusion. We set $E_{\xi}=\left(\Gamma_{\xi+1} \backslash \Gamma_{\xi}\right) \cap \Delta$. Clearly we have $\left|E_{\xi}\right|<\alpha, \bigcup_{\xi<\alpha} E_{\xi}=\Delta$. From the inductive hypothesis for every $\xi<\alpha$ there exists a partition $\left\{E_{\xi}^{n}\right\}_{n<\omega}$ of $E_{\xi}$ such that for every infinite sequence $\left\{\gamma_{n}\right\}_{n<\omega}$ in $E_{\xi}^{n} \cap \Gamma_{\sigma}$ the sequence $\left\{\pi_{\gamma_{n}} \mid K\right\}_{n<\omega}$ converges to zero.

For each $n \in \mathbf{N}$ we set $\Delta_{n}=\bigcup_{\xi<\alpha} E_{\xi}^{n}$. We show that the set $\left\{\pi_{\gamma} \mid K: \gamma \in\right.$ $\left.\Delta_{n} \cap \Gamma_{\sigma}\right\}$ contains only weakly null infinite sequences.

Assume not. Then there exists an infinite sequence $\left\{\pi_{\gamma_{n}} \mid K\right\}_{n<\omega}$ weakly converging to some $f \in C(K)$ with $f\left(x_{0}\right) \neq 0$ for some $x_{0} \in K$.

It is easy to see that the function $f$ depends only on the set $\left\{\gamma_{n}\right\}_{n<\omega}$ (i.e. for $x, y \in K$ with $x\left(\gamma_{n}\right)=y\left(\gamma_{n}\right)$ for all $n \in \omega$ we have $\left.f(x)=f(y)\right)$.

The continuity of $f$ implies that there exists a basic open neighborhood $U$ of $x_{0}$ depending on a finite subset $A$ of $\left\{Y_{K}\right\}_{K<\omega}$ so that $f(y) \neq 0$ for all $y \in U \cap K$. Notice also that for all $\xi<\alpha, E_{\xi}^{n} \cap\left\{\gamma_{n}\right\}_{n<\omega}$ is at most finite. Hence we may assume that $E_{\xi}^{n} \cap\left\{\gamma_{n}\right\}_{n<\omega}$ contains at most one element. Therefore the sequence $\left\{\gamma_{n}\right\}_{n<\omega}$ has the form $\left\{\gamma_{n}\left(\xi_{n}\right)\right\}_{n<\omega}$ for an increasing sequence $\left\{\xi_{n}\right\}_{n<\omega}$, such that

$$
E_{\xi_{1}}^{n} \cap\left\{\gamma_{n}\right\}_{n<\omega}=\left\{\gamma_{1}\left(\xi_{1}\right)\right\} .
$$

We set $n_{0}=\sup \left\{n: \gamma_{n} \in A\right\}$. Then for the element $y=x \mid \Gamma_{\xi_{n_{0}}}$ we have that $y \in U \cap K$, hence $f(y) \neq 0$. On the other hand, for every $n>n_{0}, \pi_{\gamma_{n}}(y)=0$ therefore $f(y)=\lim _{K \rightarrow \infty} \pi_{Y_{K}}(y)=0$. This is a contradiction which completes the proof of the Theorem.

REMARK. Following the arguments of the proof of the above theorem one could show the next result:

If $K$ is a compact subset of the space $\Sigma[0,1]^{\Gamma}$ and $\Delta$ is a subset of $\Gamma$ so that the set $\left\{\pi_{\gamma} \mid K: \gamma \in \Delta\right\}$ is relatively weakly compact then there exists a partition 
$\left\{\Delta_{n}\right\}_{n<\omega}$ of $\Delta$ so that for each $n \in \mathbf{N}$ and each infinite sequence $\left\{\gamma_{n}\right\}_{n<\omega}$ in $\Delta_{n}$, the sequence $\left\{\pi_{\gamma_{n}} \mid K: n \in \mathbf{N}\right\}$ is weakly null.

4. Functional analytic consequences. The main results of this section are: some characterizations of WCD (and weakly $K$-analytic) Banach spaces $E$ using operators from $E^{*}$ into $C_{1}(X)$, where $X$ is countably determined (Theorems 4.1, 4.2), a result on the strict convexity of $C_{1}(X)$ (Theorem 4.5) and some (mainly geometrical) applications of the above (Theorem 4.8).

THEOREM 4.1 (CHARACTERIZATION OF WCD BANACH SPACES). Let $E$ be a Banach space, then the following are equivalent:

(a) $E$ is a WCD Banach space.

(b) There are a nonempty subset $\Sigma^{\prime}$ of $\Sigma$ and a family $\left\{L_{K}: K \in \mathcal{K}\left(\Sigma^{\prime}\right)\right\}$ of nonempty weakly compact subsets of $E$ such that

(i) if $K \in \mathcal{K}\left(\Sigma^{\prime}\right)$ then $0 \in E$ is the only possible weak limit point of $L_{K}$;

(ii) the set $L=\bigcup\left\{L_{K}: K \in \mathcal{K}\left(\Sigma^{\prime}\right)\right\}$ is a total subset of $E$ and $L \subseteq B_{1}$; and

(iii) if $K_{1}, K_{2} \in \mathcal{K}\left(\Sigma^{\prime}\right)$ with $K_{1} \subseteq K_{2}$ then $L_{K_{1}} \subseteq L_{K_{2}}$.

(c) If $\tau=\operatorname{dim} E$ and $\alpha$ is the least cardinal such that $\tau \leq 2^{\alpha}$, then there are a nonemtpy subset $\Sigma^{\prime} \subseteq \Sigma$ and a bounded linear one-to-one operator $T: E^{*} \rightarrow$ $C_{1}\left(\Sigma^{\prime} \times\{0,1\}^{\alpha}\right)$, that is weak $k^{*}$ to pointwise continuous.

(d) There exists a bounded linear one-to-one operator $T: E^{*} \rightarrow C_{1}(Y)$, that is weak $^{*}$ to pointwise continuous, for some countably determined space $Y$.

ProOF. (a) $\Rightarrow$ (c) Let $T_{1}: C\left(B_{1}\right) \rightarrow C_{1}\left(\Sigma^{\prime} \times\{0,1\}^{\alpha}\right)$ be the operator of Theorem 2.5 and define $T_{2}: E^{*} \rightarrow C\left(B_{1}\right): T_{2}\left(x^{*}\right)(x)=x^{*}(x)$ for $x^{*} \in E^{*}$ and $x \in B_{1}$. It follows easily that $T$ is a linear isometry that is weak* to pointwise continuous. It is clear that $T=T_{1} \circ T_{2}$ has the desired properties.

(c) $\Rightarrow$ (b) For every $t \in \Sigma^{\prime} \times\{0,1\}^{\alpha}$ we set $x_{t}: E^{*} \rightarrow \mathbf{R}: x_{t}\left(x^{*}\right)=T\left(x^{*}\right)(t)$. By our assumption on $T, x_{t}$ is a weak ${ }^{*}$ continuous functional, so $x_{t} \in E$. Set for $K \in \mathcal{K}\left(\Sigma^{\prime}\right), L_{K}=\left\{x_{t}: t \in A \times\{0,1\}^{\alpha}\right\} \cup\{0\} \subseteq E$. It is clear that if $K_{1} \subseteq K_{2}$ then $L_{K_{1}} \subseteq L_{K_{2}}$, and normalizing $T$ we have $L=\bigcup\left\{L_{K}: K \in \mathcal{K}\left(\Sigma^{\prime}\right)\right\} \subseteq B_{1}$.

Since $T$ is one-to-one, the set $L$ separates points of $E^{*}$; indeed, if $x_{1}^{*} \neq x_{2}^{*}$ then $T\left(x_{1}^{*}\right) \neq T\left(x_{2}^{*}\right)$, so there is $t \in \Sigma^{\prime} \times\{0,1\}^{\alpha}$ such that $T\left(x_{1}^{*}\right)(t) \neq T\left(x_{2}^{*}\right)(t)$, i.e. $x_{t}\left(x_{1}^{*}\right) \neq x_{t}\left(x_{2}^{*}\right)$. It follows from the Hahn-Banach theorem that $L$ is a total subset of $E$.

Let $K \in \mathcal{K}\left(\Sigma^{\prime}\right)$ and $\left(x_{t_{n}}\right)_{n \in \omega}$ a sequence of distinct points of $L_{K}$. Then $x_{t_{n}} \stackrel{w}{\rightarrow}$ $0 \in E$. Indeed, if $x^{*} \in E^{*}$ then $x^{*}\left(x_{t_{n}}\right)=x_{t_{n}}\left(x^{*}\right)=T\left(x^{*}\right)\left(t_{n}\right) \rightarrow 0$, since $T\left(x^{*}\right) \in C_{1}\left(\Sigma^{\prime} \times\{0,1\}^{\alpha}\right)$ and $K$ is a compact subset of $\Sigma^{\prime}$ containing the sequence $\left(t_{n}\right)_{n \in \omega}$.

(b) $\Rightarrow$ (a) If $\Omega$ denotes the closed unit ball of $E^{*}$ with weak ${ }^{*}$ topology, then $E$ is embedded isometrically into $C(\Omega)$ and the family $\left\{L_{K}: K \in \mathcal{K}\left(\Sigma^{\prime}\right)\right\}$ consists of weakly compact subsets of $C(\Omega)$ and satisfies the conditions of Corollary 1.8 , so $C(\Omega)$ is WCD, and thus $E$ is WCD as closed linear subspace of $C(\Omega)$.

(c) $\Rightarrow$ (d) is obvious.

(d) $\Rightarrow$ (a) $E$ is embedded isometrically into $C(\Omega)$, and $\Omega$ is Gulko compact because it is homeomorphic to a pointwise compact subset of $C_{1}(Y)$ (Theorem 1.2). It follows that $E$ is WCD.

The next theorem characterizes weakly $K$-analytic Banach spaces; its proof is similar to that of Theorem 4.1. 
THEOREM 4.2. Let $E$ be a Banach space. Then the following are equivalent:

(a) $E$ is a weakly $K$-analytic Banach space.

(b) There is a family $\left\{L_{\sigma}: \sigma \in \Sigma\right\}$ of nonempty weakly compact subsets of $E$, such that

(i) if $\sigma \in \Sigma$ then $0 \in E$ is the only possible weak limit point of $L_{\sigma}$;

(ii) the set $L=\bigcup\left\{L_{\sigma}: \sigma \in \Sigma\right\}$ is a total subset of $E$ and $L \subseteq B_{1}$; and

(iii) if $\sigma, \rho \in \Sigma$ with $\sigma \leq \rho$ then $L_{\sigma} \subseteq L_{\rho}$.

(c) If $\tau=\operatorname{dim} E$ and $\alpha$ is the least cardinal such that $\tau \leq 2^{\alpha}$, then there is a bounded linear one-to-one operator $T: E^{*} \rightarrow C_{1}\left(\Sigma \times\{0,1\}^{\alpha}\right)$, that is weak $k^{*}$ to pointwise continuous.

(d) There exists a bounded linear one-to-one operator $T: E^{*} \rightarrow C_{1}(Y)$, that is weak $^{*}$ to pointwise continuous, for some $K$-analytic space $Y$.

The next theorem is known (Talagrand [21, Theorem 7.2]). We include it here because it is a direct consequence of Theorem 4.1 (see also $[\mathbf{1 8}, \mathbf{1 9}]$ ).

THEOREM 4.3. Every WCD Banach space E with the Schur property is separable.

PROOF. We recall that a Banach space $E$ has the Schur property if every weakly convergent sequence is norm-convergent. Thus the weak and norm compactness on $E$ are identical.

We consider the family $\left\{L_{K}: K \in \mathcal{K}\left(\Sigma^{\prime}\right)\right\}$ of weakly compact sets of Theorem 4.1(b). Let $L=\bigcup\left\{L_{K}: K \in \mathcal{K}\left(\Sigma^{\prime}\right)\right\}$ and define $f: L \rightarrow \mathbf{R}$ by $f(x)=\|x\|$. Since $0 \in E$ is the only possible weak limit point of each $L_{K}$, it follows from the Schur property of $E$ that $f \mid L_{K} \in C_{0}\left(L_{K}\right)$ for every $K \in \mathcal{K}\left(\Sigma^{\prime}\right)$. Now by Corollary 1.7(a), $\operatorname{supp}(f)$ is at most countable. But $\operatorname{supp}(f)=L \backslash\{0\}$ and $L$ is total. Therefore $E$ is separable.

DEFINITION 4.4. A norm $\|\cdot\|$ of a Banach space $E$ is strictly convex if for every $x, y \in E$ with $x \neq y$, such that $\|x\|=\|y\|=1$, we have $\|(x+y) / 2\|<1$.

A Banach space $E$ is strictly convexifiable if there is a strictly convex norm $\||\cdot|\|$ on $E$ equivalent to the original norm of $E$.

THEOREM 4.5. For every countably determined space $X$, the Banach space $C_{1}(X)$ admits an equivalent strictly convex norm ||$\cdot|\||$, that is pointwise lower semicontinuous (namely, for every $r>0$ the closed $r$-ball of $\left(C_{1}(X),\||\cdot|\| \mid\right)$ is a pointwise closed set).

Before the proof of this theorem we shall present some applications.

THEOREM 4.6. Let E be a WCD Banach space. Then:

(a) $E^{*}$ admits an equivalent strictly convex norm; and

(b) if $L$ is a weakly countably determined subset of a Banach space, then the Banach space $C(L)$ admits an equivalent strictly convex norm.

ProOF. (a) Let $T: E^{*} \rightarrow C_{1}\left(\Sigma^{\prime} \times\{0,1\}^{\alpha}\right)$ be the bounded linear one-to-one operator of Theorem 4.1(c). Since the space $C_{1}\left(\Sigma^{\prime} \times\{0,1\}^{\alpha}\right)$ is strictly convexifiable (see Theorem 4.5) and $T$ is one-to-one, the space $E^{*}$ must be strictly convexifiable according to a Theorem of V. Klee [7, Theorem 1, p. 100].

(b) This follows similarly using Theorem 2.5 .

REMARK Theorem 4.6(a) is known for the class of WCG Banach spaces. 
COROLLARY 4.7. There is no bounded linear one-to-one operator $T: C_{1}(\Sigma) \rightarrow$ $C_{0}(\Gamma)$, for any set $\Gamma$.

PROOF. H. Rosenthal has constructed a nonseparable closed linear subspace $E$ of the WCG space $L^{1}\left(\{0,1\}^{2^{\omega}}, \mu\right.$ ) (where $\mu$ is the Haar measure on compact group $\left.\{0,1\}^{2^{\omega}}\right)$, with an unconditional basis, which is not WCG [19, Theorem 1.1, p. 86].

Since $E$ is weakly $K$-analytic (as a closed linear subspace of a WCG Banach space), there exists according to Theorem 4.2(c), a bounded linear one-to-one operator $T_{1}: E^{*} \rightarrow C_{1}(\Sigma)$. Suppose that for some set $\Gamma$, there exists a bounded linear one-to-one operator $T_{2}: C_{1}(\Sigma) \rightarrow C_{0}(\Gamma)$. Then $T=T_{2} \circ T_{1}: E^{*} \rightarrow C_{0}(\Gamma)$ will be a bounded linear one-to-one operator. However such an operator cannot exist since $E$ has an unconditional basis and so by a theorem of W. Johnson [19, Proposition 1.3, p. 98] $E$ would be WCG.

REMARK. Since $C_{1}(\Sigma)$ is strictly convexifiable, Corollary 4.7 , implies that $C_{1}(\Sigma)$ is one more example with the properties of the examples of Dashiell and Lindenstrauss (see [6, Theorem 2 and 13, problem 8]).

We notice that the proof of the above corollary is simple but uses some nontrivial results. For another direct proof we refer to [15], where we attempt a classification of Banach spaces of the form $C_{1}(M)$ where $M$ is a separable metric space.

A much stronger result than Theorem 4.6(a) follows using the complete strength of Theorem 4.1. First, we recall that a norm $\|\cdot\|$ of a Banach space $E$ is said to be:

(a) locally uniformly convex, if for every sequence $\left(x_{n}\right)_{n \in \omega} \subseteq E$ and every $x \in E$ with $\left\|x_{y}\right\|=\|x\|=1$, the condition $\left\|\left(x_{n}+x\right) / 2\right\| \rightarrow 1$ implies that $\left\|x_{n}-x\right\| \rightarrow 0$;

(b) smooth, if for every $x \in E$ with $\|x\|=1$ there is exactly one $x^{*} \in E^{*}$ with $\left\|x^{*}\right\|=1$, such that $x^{*}(x)=1$.

THEOREM 4.8. Every WCD Banach space E admits a locally uniformly convex and smooth norm, whose dual norm on $E^{*}$ is strictly convex.

ProOF. According to Theorem 4.1(c) there exists a bounded linear one-to-one operator $T: E^{*} \rightarrow C_{1}\left(\Sigma^{\prime} \times\{0,1\}^{\alpha}\right)$ that is weak ${ }^{*}$ to pointwise continuous. Let $\||\cdot|\|$ be an equivalent strictly convex norm on space $C_{1}\left(\Sigma^{\prime} \times\{0,1\}^{\alpha}\right)$ that is pointwise lower semicontinuous.

For every $f \in E^{*}$, set \|\|$f\|\|=\|f\|+\||T(f)|\|$. It is easily seen by the above that the mapping $\|\mid \cdot\| \|: E^{*} \rightarrow[0,+\infty)$ defines an equivalent strictly convex norm on $E^{*}$ that is weak* lower semicontinuous. So by standard results [7, Theorem 3, p. 106] $E$ has an equivalent norm $\||\cdot|\|_{0}$ whose dual norm is $\|\cdot|\||$. (This norm is defined as follows: for $x \in E,\|\| x \mid \|_{0}=\sup \{|f(x)|:|\| f||| \leq 1\}$.) From a theorem of $\mathrm{V}$. Klee [7, Theorem 2(i), p. 23] we have that \|\|$\cdot\|\|_{0}$ is a smooth norm. By a theorem of Vasak [24] $E$ admits an equivalent locally uniformly convex norm $\|\mid \cdot\| \|_{1}$.

Now the conclusion follows using the techniques of Asplund [7, Corollary 1, Theorem 2, pp. 107-113].

The next corollary generalizes a result of Asplund [12, Corollary 8].

COROLlary 4.9. Let $E$ be a WCD Banach space. Then every continuous real-valued convex function $f$ defined on an open convex subset of $E$ is Gateaux differentiable in a dense $G_{\delta}$ subset of its domain of definition (that is, $E$ is a weak Asplund space, in the terminology of [12]). 
ProOF. By Theorem 4.8, any WCD Banach space $E$ admits an equivalent norm the dual norm of which is strictly convex. So Asplund's theorem [12, Theorem 7] applies to $E$.

REMARK. Using Theorem 4.8 we have a negative answer to the following problem of J. Lindenstrauss [13, problem 9]. If a Banach space $E$ admits an equivalent smooth norm, is then $E$ a subspace of a WCG Banach space? Consider a Gulko compact space $K$ that is not Eberlein compact $[\mathbf{2 0 , 2 1}]$. Then $C(K)$ is a WCD Banach space and so by Theorem 4.8 admits an equivalent smooth norm. But $C(K)$ is not isomorphic to a subspace of any WCG Banach; otherwise $K$ would be an Eberlein compact space, by a result of Benyamini, Rudin, and Wage [4].

It must be noted that Johnson and Lindenstrauss first constructed such an example [11], which is not however a WCD Banach space.

The rest of this section is devoted to the proof of Theorem 4.5. We shall use the following two lemmas.

LEMMA 4.10. Let $M$ be a separable metric space, $K$ be a compact space and $f \in l^{\infty}(M \times K)$. Then

(a) $f \in C_{1}(M \times K)$ if (and only if) $f \mid \Omega \in C_{0}(\Omega)$ for all compact subsets $\Omega$ of $M \times K$.

(b) If $f \in C_{1}(M \times K)$ then we have:

(i) the set $\{t \in M \times K: f(t) \neq 0\}$ is at most countable.

(ii) for every $t \in M \times K$ and for every $\varepsilon>0$ there exists an open set $V$ in $M$ such that $t \in V \times K$ and $f(V \times K \backslash\{t\}) \subseteq(-\varepsilon, \varepsilon)$.

ProOF. (a) Suppose that for some $\varepsilon>0$ the set $A=\{t \in M \times K:|f(t)| \geq \varepsilon\}$ is not closed and discrete in $M \times K$. Let $t=(x, y) \in A^{\prime}$ and $\left\{U_{n}: n \in \omega\right\}$ a countable neighborhood base of $x$. For every $n \in \omega$, choose $t_{n} \in A \cap\left[U_{n} \times K \backslash\left\{t, t_{1}, \ldots, t_{n-1}\right\}\right]$ where $t_{n}=\left(x_{n}, y_{n}\right), n \in \omega$. Then $x_{n} \rightarrow x$ and so $\Omega=\left(\left\{x_{n}: n<\omega\right\} \cup\{x\}\right) \times K$ is compact. Moreover $\Omega \cap A$ is infinite because $t_{n} \in \Omega \cap A, n \in \omega$, and the proof of claim (a) is complete.

(b) (i) We have $\{t \in M \times K: f(t) \neq 0\}=\bigcup_{n \in \omega}\{t \in M \times K:|f(t)| \geq 1 / n\}$ where each set in the right side is countable as a closed and discrete subset of the Lindelöf space $M \times K$.

(ii) Assume that the assertion is not valid and let $t=(x, y) \in M \times K$ such that for some $\varepsilon>0, f(V \times K \backslash\{t\}) \nsubseteq(-\varepsilon, \varepsilon)$ for every open set $V$ in $M$ such that $t \in V \times K$. Then we can easily find a sequence $t_{n}=\left(x_{n}, y_{n}\right), n \in \omega$, such that $t_{0}=t, t_{n} \notin\left\{t_{0}, t_{1}, \ldots, t_{n-1}\right\}, x_{n} \rightarrow x$ and $\left|f\left(t_{n}\right)\right| \geq \varepsilon$. We set $A=\left\{x_{n}: n<\right.$ $\omega\} \cup\{x\}$ and observe that $\left\{t_{n}: n<\omega\right\}$ is an infinite subset of the compact $A \times K$ and $\left|f\left(t_{n}\right)\right| \geq \varepsilon$ for $n<\omega$, a contradiction.

LEMMA 4.11. For every countably determined space $X$ there exist a separable metric space $M$, a compact space $K$ and a linear isometry $T: C_{1}(X) \rightarrow C_{1}(M \times K)$ that is continuous for the topology of pointwise convergence.

PROOF. Let $F$ be a closed subset of a product $M \times K$, where $M$ is a separable metric space and $K$ a compact space, such that there exists $f: F \rightarrow X$ continuous onto. Choose $Z \subseteq F$ such that $f \mid Z$ is one-to-one and $f(Z)=X$. Then for every $A \subseteq X$ with $A^{\prime}=\varnothing$, we have $\left(f^{-1}(A) \cap Z\right)^{\prime}=\varnothing$. Indeed, let $\Omega$ be a compact subset of $\bar{M} \times K$ and set $B=f^{-1}(A) \cap Z$. Then $f(B \cap \Omega)$ is a relatively compact subset 
of $A$ and so $|B \cap \Omega|=|f(B \cap \Omega)|<\omega$. Thus by Lemma 4.10(a), $\chi_{B} \in C_{1}(M \times K)$, that is, $B^{\prime}=\varnothing$.

Now, by the above, the mapping

$$
T: C_{1}(X) \rightarrow C_{1}(M \times K) \quad \text { with } T(g)(t)= \begin{cases}0, & t \notin Z, \\ g(f(t)), & t \in Z,\end{cases}
$$

has the desired properties.

PROOF OF THEOREM 4.5. Using the fact that strict convexity is preserved by closed linear subspaces, it suffices by Lemma 4.11 to prove the theorem when $X=M \times K$.

For an infinite set $\Gamma$ and for every $f \in l^{\infty}(\Gamma)$ we set

$$
D(f)=\sup \left\{\left[\sum_{n=1}^{\infty} \frac{1}{4^{n}}\left|f\left(S_{n}\right)\right|^{2}\right]^{1 / 2}:\left(S_{n}\right)_{n \in \omega} \subseteq \Gamma \text { one-to-one sequence }\right\} .
$$

It is an easy (and known) fact that this formula defines a norm equivalent with the supremum norm of $l^{\infty}(\Gamma)$.

Let $\left(U_{n}\right)_{n \in \omega}$ be a countable base for the topology of $M$ with $U_{0}=M$. Set $G_{n}=$ $U_{n} \times K, n=0,1,2, \ldots$ For every $f \in C_{1}(M \times K)$ we set $\varphi_{n}(f)=D\left(f \mid G_{n}\right), n=$ $0,1,2, \ldots$ and

$$
N_{1}(f)=\left[\sum_{j=0}^{\infty}\left(\frac{1}{4}\right)^{j} \varphi_{j}(f)^{2}\right]^{1 / 2} .
$$

It is not hard to see that the mapping $N_{1}: C_{1}(M \times K) \rightarrow \mathbf{R}$ is a pointwise lower semicontinuous norm on $C_{1}(M \times K)$ equivalent with the supremum norm.

We shall prove that $N_{1}$ is a strictly convex norm. For this purpose, let $f, g \in$ $C_{1}(M \times K)$ such that $N_{1}(f)=N_{1}(g)$ and $N_{1}(f+g)=N_{1}(f)+N_{1}(g)$. It is enough to prove that $f=g$ (see Definition 4.4). We have that

$$
\begin{aligned}
N_{1}(f+g) & =\left\|\left\{\left(\frac{1}{2}\right)^{j} \varphi_{j}(f+g)\right\}\right\|_{2} \leq\left\|\left\{\left(\frac{1}{2}\right)^{j} \varphi_{j}(f)+\left(\frac{1}{2}\right)^{j} \varphi_{j}(g)\right\}\right\|_{2} \\
& \leq\left\|\left\{\left(\frac{1}{2}\right)^{j} \varphi_{j}(f)\right\}\right\|_{2}+\left\|\left\{\left(\frac{1}{2}\right)^{j} \varphi_{j}(g)\right\}\right\|_{2} \\
& =N_{1}(f)+N_{1}(g)=N_{1}(f+g),
\end{aligned}
$$

so we have equalities. It follows from the strict convexity of the $l_{2}$-norm that

$$
\left\{\varphi_{j}(f+g)=\varphi_{j}(f)+\varphi_{j}(g) \text { and } \varphi_{j}(f)=\varphi_{j}(g), j=0,1,2, \ldots\right\} .
$$

Let $t \in M \times K$ and suppose that $f(t) \neq 0$ (the proof is similar when $g(t) \neq 0$ ). It follows immediately from Lemma 4.10(b)(ii) that there exists $n_{0} \in \omega$ such that:

$$
|f(t)|>\sup \left\{|f(s)|: s \in G_{n_{0}} \backslash\{t\}\right\} .
$$

Now the conclusion follows immediately from (1) and (2) in connection with following lemma of Dashiell-Lindenstrauss [6], where $\Gamma=G_{n_{0}}$.

LEMMA. Let $\Gamma$ be a nonempty set and $f, g \in l^{\infty}(\Gamma)$ such that $D(f)=D(g)$ and $D(f+g)=D(f)+D(g)$. Then $f(t)=g(t)$ for all $t \in \Gamma$ with

$$
|f(t)|>\sup \{\mid f(s): s \in \Gamma \backslash\{t\}\}
$$

or

$$
|g(t)|>\sup \{|g(s)|: s \in \Gamma \backslash\{t\}\} .
$$


REMARK. The proof of Theorem 4.5 uses a technique of Dashiell-Lindenstrauss (see Theorem 10 of $[6]$ ).

NOTE ADDED IN PROOF. Recently Professor Roman Pol has kindly informed me that characterizations of Gulko and Talagrand compact spaces closely related to Theorem 3.3, were obtained independently by G. A. Sokolov, On some classes of compact spaces lying in $\Sigma$-products, Comment. Math. Univ. Carolin. 25 (1984), 219-231.

\section{REFERENCES}

1. D. Amir and J. Lindenstrauss, The structure of weakly compact sets in Banach spaces, Ann. of Math. (2) 88 (1968), 35-46.

2. S. Argyros, S. Mercourakis and S. Negrepontis, Analytic properties of Corson compact spaces, Proc. Fifth Prague Topological Sympos. (August 1981), Heldermann-Verlag, Berlin, 1982, pp. 12-23.

3. S. Argyros and S. Negrepontis, On weakly K-countably determined spaces of continuous functions, Proc. Amer. Math. Soc. 87 (1983), 731-736.

4. Y. Benyamini, M. E. Rudin and M. Wage, Continuous images of weakly compact subsets of Banach spaces, Pacific J. Math. 70 (1977), 309-324.

5. J. P. R. Christensen, Topology and Borel structure, North-Holland Math. Studies, no. 10, North-Holland, Amsterdam, 1974.

6. F. K. Dashiell and J. Lindenstrauss, Some examples concerning strictly convex norms on $C(K)$ spaces, Israel J. Math. 16 (1973), 329-342.

7. J. Diestel, Geometry of Banach spaces, Selected topics, Lecture Notes in Math., vol. 485, Springer-Verlag, Berlin, 1975.

8. R. Engelking, General topology, PWN, Warsaw, 1977.

9. S. P. Gulko, On the structure of spaces of continuous functions and their complete paracompactness, Russian Math. Surveys 34 (1979), 36-44 =Uspekhi Mat. Nauk 34 (1979), 33-40.

10. G. Gruenhage, A note on Gul'ko compact spaces, preprint.

11. W. Johnson and J. Lindenstrauss, Some remarks on W.C.G. Banach spaces, Israel J. Math. 17 (1974), 219-230.

12. D. G. Larman and R. R. Phelps, Gateaux differentiability of convex functions on Banach spaces, J. London Math. Soc. (2) 20 (1979), 115-127.

13. J. Lindenstrauss, Weakly compact sets-their topological properties and the Banach spaces they generate, Ann. of Math. Studies, no. 69, Princeton Univ. Press, Princeton, N. J., 1972, 235-273.

14. S. Mercourakis, Corson-compact spaces and the structure of $K$-analytic Banach spaces, Doctoral dissertation, Athens Univ., 1983 (Greek).

15. __ Some characterizations of analytic metric spaces, Pacific J. Math. (to appear).

16. S. Negrepontis, Banach spaces and topology, Handbook of Set-Theoretic Topology (K. Kunen and J. Vaughan, eds.), North-Holland, Amsterdam, 1984, pp. 1041-1138.

17. C. A. Rogers and J. E. Jayne, $K$-analytic sets, Analytic Sets, Academic Press, London, 1980.

18. H. P. Rosenthal, On injective Banach spaces and the spaces $L^{\infty}(\mu)$ for finite measures $\mu$, Acta Math. 124 (1970), 205-248.

19. Math. 28 (1974), 83-111.

20. __ Point-wise compact subsets of the first Baire class, Amer. J. Math. 99 (1977), 362-378. 
21. M. Talagrand, Espaces de Banach faiblement $K$-analytiques, Ann. of Math. (2) 110 (1979), 407-438.

22. __ Deux generalisations d'un théorème de I. Namioka, Pacific J. Math. 81 (1979), 239-251.

23. _ A new countably determined Banach space, Israel J. Math. 47 (1984), 75-80.

24. L. Vasak, On one generalization of weakly compactly generated Banach spaces, Studia Math. 70 (1980), 11-19.

Section of Mathematical Analysis, Athens University, Panepistemiopolis, 15781 ATHENS, GREECE 